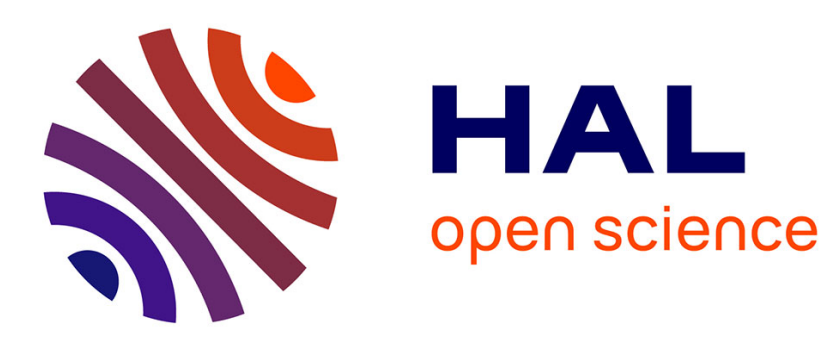

\title{
Temperature dependence of the structure and electrostatics of Newton Black Films: insights from computer simulations
}

Fernando Bresme, Jordi Faraudo

\section{- To cite this version:}

Fernando Bresme, Jordi Faraudo. Temperature dependence of the structure and electrostatics of Newton Black Films: insights from computer simulations. Molecular Simulation, 2007, 32 (12-13), pp.1103-1112. 10.1080/08927020600815337 . hal-00514982

\section{HAL Id: hal-00514982 \\ https://hal.science/hal-00514982}

Submitted on 4 Sep 2010

HAL is a multi-disciplinary open access archive for the deposit and dissemination of scientific research documents, whether they are published or not. The documents may come from teaching and research institutions in France or abroad, or from public or private research centers.
L'archive ouverte pluridisciplinaire HAL, est destinée au dépôt et à la diffusion de documents scientifiques de niveau recherche, publiés ou non, émanant des établissements d'enseignement et de recherche français ou étrangers, des laboratoires publics ou privés. 


\section{Molecular Simulation \\ Journal of \\ Experimental Nanoscience \\ - Taylor \& Francis}

\section{Temperature dependence of the structure and electrostatics of Newton Black Films: insights from computer simulations}

\begin{tabular}{|r|l|}
\hline Journal: & Molecular Simulation/Journal of Experimental Nanoscience \\
\hline Manuscript ID: & GMOS-2006-0067 \\
\hline Journal: & Molecular Simulation \\
\hline Date Submitted by the \\
Author: & $26-$ Apr-2006 \\
\hline $\begin{array}{r}\text { Complete List of Authors: } \\
\text { Keywords: }\end{array}$ & $\begin{array}{l}\text { Bresme, Fernando; Imperial College London, Chemistry } \\
\text { Faraudo, Jordi; Universidad Autonoma de Barcelona, Physics }\end{array}$ \\
\hline &
\end{tabular}

\section{SCHOLARONE Manuscripts}




\title{
Temperature dependence of the structure and electrostatics of Newton Black Films: insights from computer simulations
}

\author{
Fernando Bresme ${ }^{1}$ and Jordi Faraudo ${ }^{2}$ \\ (1) Department of Chemistry, Imperial College London, \\ Exhibition Road, London, SW7 2AZ, United Kingdom. \\ (2) Department de Fisica, Universitat Autonoma de Barcelona, \\ Facultat de Ciencies, E-08193 Bellaterra, Spain
}

(Dated: April 26, 2006)

\begin{abstract}
Newton Black Films (NBF) can be obtained from solutions containing ionic surfactants. These thin structures have typical thickness of the order of 3 nanometers and are similar to biological membranes. An understanding of the NBF structure and stability is of relevance to problems concerned with the biophysics of the cell. From a practical point of view the stability of NBFs determines the stability of dispersions and foams, which are relevant in a number of industrial areas such as foodstuffs, cosmetics or distillation. In this paper we investigate the temperature dependence of the structure and electrostatics of sodium dodecyl sulfate Newton Black Films using large scale computer simulations. We show that the film roughness does not change monotonically with temperature. Cooling a film initially at ambient temperature does not result in a significant change of the film roughness, whereas heating results in a dramatic change of the roughness. This result suggest that the film roughnes cannot be explained in terms of thermally activated fluctuations alone. Notably, all these structural changes occur without a significant modification of the total thickness of the film. The degree of polarization of the water molecules and the electrostatic fields in the NBF also show a very weak dependence on temperature, suggesting that water remains strongly ordered in the NBF even at high temperatures $(\approx 350 \mathrm{~K})$. We conclude that the anomalous dielectric response observed in NBF at ambient temperature should also be present at high temperatures.
\end{abstract}




\section{INTRODUCTION}

Black Films can be formed from solutions of ionic surfactants, such as sodium dodecyl sulfate, by variation of electrolyte concentration and/or the temperature. In this way Black Films of different thicknesses can be obtained; Common Black Films (CBF), with thicknesses of the order of tens of nanometers (5-100 nm), and Newton Black Films (NBF), extremely thin structures with characteristic thickness of the order of $3 \mathrm{~nm}$. Black Films are too thin to reflect incident light and as consequence they appear as black spots. Newton Black Films were first described by Newton and Hooke in the $17^{t h}-18^{t} h$ century. A more quantitative account of these films, both theoretical and experimental, was provided by Perrin and Wells at the beginning of the $20^{t} h$ century. Perrin already figured out the possible structure of these films, consisting of surfactant layers and a core characterized by the absence of free water. Perrin's intuition corresponds rather well with our current understanding of the structure of Newton Black Films.

Black Films are very relevant in a number of scientific and industrial problems. They have played a very important role in the development of theoretical approximations to interpret surface and colloid forces [1]. Also the structure of Black Films, similar to the membranes that encapsulate all living cells, makes them interesting experimental models to investigate questions of relevance to the biophysics of the cell. On the practical side, since Black Films do appear as transient structures in the coalescence of liquid-liquid dispersions (nano and microemulsions) and air-liquid dispersions (foams), they are relevant in a number of industrial areas; foodstuffs, cosmetics, distillation and laundering, for instance. A major objective in modern studies of Black Films is the understanding of their stability. This information is essential to advance our understanding of the stability of foams and emulsions for instance [2-5], and answer practical questions concerned with the ability of some chemicals to generate persistent emulsions or foams.

Recent experiments have provided a more clearer picture of the transitions and structure of Black Films. The transition from Common Black Film to Newton Black Film can be induced by changing the ionic strength of the medium and/or the temperature [7-9]. Common Black Films appear normally at low electrolyte concentrations and high temperatures. It is observed that the thickness of the CBF depends on the electrolyte concentration, a feature that is consistent with the DLVO theory [2], which describes the interactions in these films as 
a balance of van der Waals and double layer interactions. On the other hand, the thickness of Newton Black Films is essentially independent on the electrolyte concentration, the disjoining pressure, and even it shows to be insensitive to small changes of temperature [10]. These observations, in particular the insensitivity of the thickness to double layer forces, points strongly toward the existence of forces that depart from the classical DLVO interpretation. The analysis of disjoining pressure isotherms in some CBF and NBF films (particularly in the case of SDS films) reveals clear deviations from the predictions of the DLVO theory $[3,6]$. Thus, a major challenge in the investigation of thin films is to provide a microscopic interpretation of the origin of the forces that stabilize Newton Black Films. This problem has motivated a large number of works in the area. In particular experiments on a number of systems, from phospholipid bilayers [11] to DNA [12], have shown that charged surface in close contact interact according to a strongly repulsive exponential force, whose decay length is of the order to the water molecule diameter (1-3 $\AA$ ). This force is normally referred to in the literature as hydration force. There have been two major theoretical breakthroughs that have provided an explanation to this force. One of them is based on the idea that the water confined between polar and charged surfaces is strongly polarized. Exploiting this idea, Marcelja and Radic developed a simple and elegant theory that predicts a repulsive force with the right exponential decay [13]. More recently, further theoretical developments beyond the DLVO theory have also included the polarization of water as essential ingredient of the theory [14]. The relationship between the hydration force and water polarization has been recently questioned by Israelachvili and Wennerstrom [15]. It has been suggested that there is no evidence for a significant structure of water near interfaces [16]. These authors advocated entropic forces as the origin of the short range force that stabilize membranes. The rational behind this idea is that thin films are flexible objects that can undergo thermal fluctuations (protrusions, undulations), which result in a repulsive force of entropic origin. This idea is very appealing, but it has received some criticism [17]. It is clear that a complete answer to the origin of the hydration forces, and hence, to the stability of Black Films is still far from complete.

In order to investigate the molecular mechanisms leading to the stability of Black Films, it would be desirable to gain a deep molecular level understanding of the structure and behavior of these systems. Very recently, X-Ray [7] and neutron scattering [10] experiments have provided important information in this direction, reporting accurate results on the thickness 
of Newton Black Films and its dependence on electrolyte concentration and temperature. Further insight on the microscopic structure of thin films and its relation to precise intermolecular forces can be attained using computer simulations. Gamba et. al. [18] reported one of the first attempts to simulate a Newton Black Film at conditions compatible with experiments. These simulations provided important information on the structure of the film, and pointed out the slow dynamics of the film surfactants. Recently we have investigated sodium dodecyl sulfate Newton Black Films using large scale computer simulations [19]. We found that indeed the dynamics is very slow. This makes necessary to use long simulations in order to obtain equilibrium states. These long simulations enabled us to investigate in detail the electrostatics of Newton Black Films. We showed that the polarization and the electric field are not proportional to each other. In fact, no local relation can be established between the local value of the total electric field and the electrostatic polarization [20]. Hence, a local dielectric permittivity must be defined in this system. This anomalous dielectric behavior of water in the Black Films results in a nonclassical electrostatic repulsive force that is compatible with the strong repulsive force reported in the experiments. We have suggested that the anomalous dielectric behavior provides the microscopic mechanism that explains the origin of the controversial 'hydration force' [21].

In this paper we investigate the influence of temperature on the structure and electrostatics of sodium dodecyl sulfate Newton Black Films. A knowledge of the relationship between the temperature and the properties of these films is relevant to understand the role played by the temperature in Common to Newton Black Films transitions, as well as to address the overall effect of thermal fluctuations on the film structure.

\section{COMPUTATIONAL MODEL OF SODIUM DODECYL SULFATE NEWTON BLACK FILMS}

The Newton Black Film we wish to investigate consists of a thin water layer coated with surfactants, sodium dodecyl sulfate (SDS). The water core contains the counterions, $\mathrm{Na}^{+}$, resulting from the dissociation of the surfactants (cf. Figure 1). X-Ray reflectivity experiments show that the thickness of the equilibrium SDS Newton Black Film is insensitive to the electrolyte concentration [7]. This suggests that the only ions present in the film are the counterions. Therefore in our simulations we consider free salt conditions. 
Our model considers explicitly the surfactants, the counterions and water. The molecular nature of the solvent is very often neglected in the theoretical investigations of these films, particularly in theoretical approaches based on the DLVO theory. Nonetheless, molecular effects become significant in these thin films, and can strongly influence the dielectric response of the solvent [20]. Therefore water must be considered explicitly. In our model the surfactant hydrocarbon chain is represented using a united atom approach (see Figure 2), in which the $\mathrm{CH}_{2}$ and $\mathrm{CH}_{3}$ groups are substituted by a pseudoatom. This model is computationally less demanding than a full atom model, since the surfactant is represented with 17 atoms instead of the 42 needed in the full atom case. Otherwise the head group is represented explicitly. The surfactant intramolecular interactions were modeled using as starting point the AMBER forcefield [23]. Bonding and bending degrees of freedom were handled using harmonic potentials,

$$
\begin{aligned}
U_{\text {bond }}\left(r_{i j}\right) & =\frac{k_{i j}}{2}\left(r_{i j}-r_{e}\right)^{2} \\
U_{\text {bend }}\left(\theta_{i j k}\right) & =\frac{k_{i j k}}{2}\left(\theta_{i j k}-\theta_{e}\right)^{2}
\end{aligned}
$$

where $r_{i j}$ represent the distance between atoms $i j$, and $r_{e}$ and $k_{i j}$ represent the equilibrium distance and force constant for the bonding potential respectively. Similarly, $\theta_{i j k}$, represents the angle determined by atoms $i j k$, and $\theta_{e}$ and $k_{i j k}$ are the equilibrium angle and force constant of the bending potential. The chain conformation is constrained through a torsional potential defined as:

$$
U_{t o r}\left(\phi_{i j k l}\right)=A_{i j k l}\left[1+\cos \left(m \phi_{i j k l}+\delta\right)\right]
$$

where $\phi_{i j k l}$ is the dihedral angle defined by four consecutive atoms, $i j k l$, in the surfactant chain, and following previous works $[19,24]$ we set $m=3$ and $\delta=0$. Table I contains detailed information on the parameters employed to model the SDS Newton Black Film.

In addition to the intramolecular bonds, 'non-bonded' atoms interact one each other through van der Waals interactions modeled using the Lennard-Jones potential. Values for the interaction strength and atom diameter are collected in Table II.

The negative charge in the surfactant head group was represented using a charge distribution based on semiempirical quantum mechanical computations [25]. Details on the 
location and magnitude of the partial charges are given in Figure 2 and Table II. Finally the surfactant counterion, $\mathrm{Na}^{+}$, was modeled as a Lennard-Jones charged sphere (see Table II for details). The cross interactions between atoms of different species were obtained from the data given in Table II using the combining rules: $\sigma_{i j}=\left(\sigma_{i}+\sigma_{j}\right) / 2$ and $\epsilon_{i j}=\sqrt{\epsilon_{i} \epsilon_{j}}$. All the Lennard-Jones interactions were cutoff at $14 \AA$.

One important element in the simulation of Newton Black Films is the water model. We have chosen the popular Simple Point Charge/Extended SPC/E model [26] (see Table II), which is appropriate in the study of atomic-level simulations of solutions and biomolecular systems[30]. It has been found that the SPC/E rigid model can be successfully used to investigate hydrophobic [27] and charged surfaces [28], yielding very similar results to those obtained with more involved polarizable models. These studies show that the water structure and the associated electrostatic fields are relatively independent on the water polarizability.

The Coulombic interactions arising from the partial charges in the surfactants, water and the counterion charge, were computed using the three dimensional (3D) version of the Ewald summation method. We note that all our simulations are performed using simulation boxes that are fully periodic in the three directions, whereas the NBF is periodic in 2 directions only. The 3D Ewald method has to be applied with some care when considering interfacial systems that are truly periodic in two dimensions only as it is the case here. Using the 3D Ewald method, the film has an unrealistic interaction with its images in the 'non periodic' direction, $z$ in our case. This interaction is proportional to the $z$ component of the dipole moment of the film. Due to symmetry of our system, the dipole is zero and consequently the residual interaction is also zero. In any case in order to ensure proper convergence in the computation of the electrostatic interactions it is advisable to elongate the box in the non periodic direction in order to minimize artificial effects [29]. $\mathrm{L}_{z} \approx 2 L_{x}=2 L_{y}$, where $L_{x}$ and $L_{y}$ are the length of the simulation box in the film plane, represents an adequate choice to simulate our Newton Black Films (see below). Finally the Ewald parameters were set to ensure that the relative error in the computation of the Coulombic interactions was not larger than $\approx 10^{-6}$.

The initial configuration was prepared as follows. We generated a two dimensional lattice of surfactants arranged in a simple square lattice. This monolayer was replicated to create a bilayer. The surface area was set to match experimental estimates of the surface area per surfactant, $33 \AA^{2}[7]$. For a system consisting of 64 surfactants per monolayer this result 
in a simulation box with dimension in the film plane of $L_{x}=L_{y}=46 \AA$. The sodium ions were distributed either randomly or in a lattice in the core of the film. Finally the film core was filled with a preequilibrated sample of SPC/E water. Since the simulation box is fully periodic, in order to model the film which is periodic only in two dimensions, we elongated the simulation box in the $\mathrm{z}$ direction in order to create vacuum regions around the film and minimize the interactions of the film with its images (see Ewald summation discussion above). The length of the box in the $\mathrm{z}$ direction was set to $L_{z}=100-120 \AA$. For this simulation setup the pressure normal to the film is determined by the water vapor pressure in the film. This pressure is negligible at ambient temperature and the normal pressure is essentially zero.

The simulations were performed in the canonical ensemble (NVT) using the Nose-Hoover thermostat, with a relaxation constant of $0.5 \mathrm{ps}$, and the trajectories were integrated using a time step of 2 femtoseconds. Newton Black Films exhibit a slow dynamics. Typically the diffusion coefficient of the dodecyl sulfate anion is $\approx 0.02 \AA^{2} / p s$ [19], i.e., one order of magnitude smaller than that of bulk water. Consequently rather long equilibration periods are necessary in order for the film to relax to the equilibrium thickness. A typical simulation involved 1 nanosecond equilibration and at least one further nanosecond of production time.

The typical system size for Newton Black Films can go from a few thousands of atoms up to several tens of thousand of atoms. As a result, the simulation can become rather computationally demanding. Therefore, we run our simulations in supercomputers using the parallel code DLPOLY 2 [22]. Figure 3 shows an example of the scaling of the computations with the number of processors for two films with different water content (4.4 water molecules per surfactant - 3981 particles - and 44 water molecules per surfactant - 19296 particles). We find that DLPOLY shows a good scaling with the number of processors. Production runs were performed using 32 processors for small films and 128 processors for thick films. In this paper we focus on the properties of small systems, 128 surfactants, 128 counterions and 559 water molecules. The thickness of this film corresponds with the one measured in the experiments [7]. 


\section{STRUCTURAL PROPERTIES: DEPENDENCE WITH TEMPERATURE}

The temperature can strongly influence the properties of thin films. In particular it can be used as a variable to induce Common to Newton Black Film transitions. It has been observed that high temperature favor Common Black Films whereas Newton Black Films are more stable at low temperatures. We discuss in the following the effect of the temperature in the structure of sodium dodecyl sulfate Newton Black Films (SDS-NBF). Comparison of the computed and experimental X-Ray density profiles [7], suggests that the equilibrium structure spontaneously formed in the experiments corresponds to a film with a water content of the order of 4 water molecules per surfactant $[18,19]$. Therefore we have focused our investigation on a film (4.4 water molecules per surfactant) whose thickness that shows good agreement with the experiments. We note that the thickness of the film is quite sensitive to the water content [19]. It changes linearly with the number of water molecules for systems with more than 2.5 water molecules per surfactant. This linear dependence is compatible with the behavior expected for an incompressible fluid. We estimated that $\approx 2$ water molecules per surfactant are part of the solvation structure of surfactant layers [19]. The system we investigate here, 4.4 water molecules / surfactant, is in the linear regime, but very close to the region where no free water exist inside the film core. This structure is compatible with the model outlined by Perrin and with more recent experimental investigations using X-Ray reflectivity [7].

Figure 4-a shows a typical snapshot of the SDS Newton Black Films at 298 K. Although the surfactant layers are very close one each other, one can still appreciate two well defined layers with a small gap in between. Most of the counterions are adsorbed at the layers. Indeed we estimated in our previous work [19] that only $\approx 10 \%$ of the surfactants are fully dissociated. The number of dissociated surfactants remains essentially constant thorugh the simulations, with small fluctuations as expected in the equilibrium state. We observe that the surfactant ionization rate is of the order of $\sim 3$ ions per picosecond. This rate is compensated by an equal rate of adsorption of $\mathrm{Na}^{+}$counterions. This results in a small amount of free counterions in the film core (cf. Figure 4-a).

The film structure can be quantified by computing the density profile across the film (cf. Figure 5-a). The profiles show that the surfactant layers are well defined (see S profiles in Figure 5-a). There is very little overlap between the layers in the middle of the film. 
The sodium counterions follow essentially the head group profile. This result contrast with traditional statistical-mechanics models [32] which assume that adsorbed counterions form a so-called Stern layer, i.e., a layer of counterions adjacent to the surfactant plane in the inner part of the film (see for example Figure 1 in [32]). In fact our results show that the adsorbed counterions belong to the same surface defined by the surfactant layers.

In order to investigate the impact of temperature on the NBF structure, we have considered two additional temperatures, HIGH, corresponding to $348 \mathrm{~K}$ and, LOW, $248 \mathrm{~K}$, i.e., $\pm 50 \mathrm{~K}$ about ambient temperature. It is worth pointing out that experiments have considered in the past temperatures of the order of 50 degrees Celsius. In comparison our HIGH temperature is on the upper end of what can be expected from an experiment, but we expect this will enable us to describe more clearly the structural changes of the film. On the other hand the LOW temperatue, is above the melting temperature of the SPC/E model (216 $\mathrm{K}$ at 1 bar pressure) [31].

First we note that lowering the temperature from $298 \mathrm{~K}$ to $248 \mathrm{~K}$ induces very little changes in the density profiles. Indeed, the LOW temperature head group profiles show two well defined peaks with a width similar to that of the ambient temperature system. We note that thermal fluctuations are reduced upon a reduction of temperature but this does not seem to be reflected in the structure of the film as described by the density profiles. The HIGH temperature case does show clear differences though. In this case there is a large increase in the width of the profiles, which is consistent with the expected effect of the thermal fluctuations. The surface layers appear much less well defined, and the minimum in the sodium profile disappears, suggesting that the two layer almost fuse together, to the extent that one cannot assign the counterions to one layer or another. The changes observed in the density profiles suggest a large modification of the inner structure of the HIGH temperature film. Fig 4-b provides further insight on these changes, showing significant fluctuations of the surfactant positions around the film plane. One can observe in the middle of the film a region where the surfactants are clearly in contact. These changes in the film structure are also reflected in the water density profiles (cf. Fig. 5-b), which shows that increasing the temperature results in a reduction in the amount of water inside the film with respect to that found at ambient and LOW temperature conditions. Again in the last two cases the water density profiles are insensitive to a change in temperature. It is remarkable that all these structural changes take place without a noticeable change in the overall thickness of 
the film. In fact if one estimates this thickness from the distance between the maxima in the $\mathrm{CH}_{3}$ density profiles (cf. Fig. 5a), one finds that the thickness is essentially constant. Recent neutron scattering experiments have investigated the dependence of the thickness of Newton Black Films formed from oil-SDS-water dispersions as a function of temperature [10]. Interestingly these authors found that the film thickness is rather insensitive to temperature changes. This seems to agree with the general behavior observed here, but it is important to note, that this insensitivity does not imply that the film structure is the same. In fact our results show that the internal structure of the film may change very much in going from ambient to HIGH temperature conditions.

The roughness function offers a complementary approach to investigate in more detail the fluctuations undergone by the NBFs with temperature. Essentially, the roughness function quantifies the out of plane fluctuations of the Newton Black Films and can be computed through the height-height correlation function:

$$
\zeta(|\vec{R}|)=\sqrt{\left\langle[z(\vec{r})-z(\vec{r}+\vec{R})]^{2}\right\rangle}
$$

where $z(\vec{r})$ and $z(\vec{r}+\vec{R})$ represent the $\mathrm{z}$ coordinate of two $\mathrm{S}$ atoms on the same monolayer lying a distance $\mathrm{R}$ one each other. We showed in our previous work that the roughness obtained from this method shows a very a good agreement with the experimental estimates [19]. We note that, in these ultra-thin films, it becomes problematic to assign a given $\mathrm{S}$ atom to a particular monolayer. In order to identify the $\mathrm{S}$ atoms pertaining to the same monolayer, we checked that their corresponding terminal groups $\left(\mathrm{CH}_{3}\right)$ were lying on the same part of the monolayer, i.e., upper or lower part. Figure 6 depicts the roughness function for the three temperatures investigated in this work. The limiting value of $\zeta$ for large $R$, quantifies the average roughness of the monolayers. Our data show (cf. Fig. 6) that the temperature reduction, LOW, does not result in an overall decrease of the film roughness, as might be expected in terms of thermal fluctuations. In fact we observe essentially the same roughness within statistical error. On the other hand the roughness function of the HIGH temperature system shows a dramatic increase, the roughness being almost twice as large as the roughness of the ambient temperature system. Increasing the temperature is expected to increase the thermal fluctuations of the thin film and hence the roughness. According to the capillary waves theory, the interfacial fluctuations should increase as the ratio of thermal energy to the surface tension $k_{B} T / \gamma$. If the film roughness would increase only on the bases 
of capillary fluctuations we would expect the roughness to increase according to the ratio:

$$
\frac{\zeta_{2}}{\zeta_{1}} \propto \sqrt{\frac{T_{2} \gamma_{1}}{T_{1} \gamma_{2}}}
$$

The roughness of the HIGH temperature system increases by a factor of $3 / 2$ (we estimate the roughness for ambient and HIGH temperature systems from the limiting values of $\zeta$ in Figure 6). This would imply that the surface tension of the HIGH temperature system should decrease at least by a factor of 2 , which could well be within the expected change in surface tension. Obviously checking whether thermal fluctuations explain the increase in the roughness would require further investigations, in particular the computation of the film surface tension, which is out of the scope of this paper. We note that we cannot use a similar argument to explain the observations relative to the LOW temperature system, since despite the fact the thermal energy changes, and therefore the thermal fluctuations should be reduce, we observe essentially the same film roughness. This suggests that thermal fluctuations might not be the only element defining the roughness and therefore the disorder of the film.

We have shown that the temperature has a dramatic effect on the structure of HIGH temperature systems. It is instructive to analyze how all these changes would appear in an experimentally measurable quantity such as the electron density profile. Figure 7 shows the electron density profile, computed as the number of electrons per unit volume across the Newton Black Film. We have also included the experimental profile [7]. Again the profiles for ambient and LOW temperature systems are very similar, showing the insensitivity of this property to the temperature reduction. The HIGH temperature system is noticeable different though, with no neat resolution of the two central peaks of the profile, indicating that the structural changes described here should be clearly seen in an experiment. Again, if we consider the whole profile to estimate the thickness of the NBF, we would conclude that the thickness is insensitive to temperature, despite the important structural changes undergone by the film at HIGH temperatures. 


\section{TEMPERATURE DEPENDENCE OF WATER POLARIZATION AND ELEC- TROSTATICS OF NEWTON BLACK FILMS}

In the following we investigate the influence of the temperature on the water polarization and electrostatic properties of the Newton Black Films. Again we focus on the system corresponding to 4.4 water molecules per surfactant. Water polarization has been discussed in the past as the possible origin of the hydration force that stabilize charged bilayers. Marcelja and Radic included water orientation as the order parameter in their Landau expansion of the free energy [13]. Therefore this quantity is of particular significance in the investigation of thin films. The orientation of the water dipole moments with respect to the film plane can be computed through the ensemble average:

$$
\langle\cos (\theta)\rangle=\left\langle\frac{\vec{\omega} \cdot \vec{z}}{|\vec{\omega}| \cdot|\vec{z}|}\right\rangle
$$

where $\theta$ ) is the angle between the water molecule dipole moment vector, $\vec{\omega}$, and the vector normal to the bilayer plane, $\vec{z}$. Figure 8 shows the variation of the dipole moment orientation for the three temperatures considered in this work. Our result indicates that for this small film, water molecules are polarized with the dipole moment pointing slightly toward the aliphatic chains. This conformation is compatible with one in which one of the hydrogen of the water molecule is pointing toward the hydrocarbon region. A similar structure has been observed in water next to hydrophobic surfaces [33]. In the NBF though, the water orientation results from the interactions of water with the charged species (ionic surfactants and counterions) as well as with the hydrophobic part of the film, i.e., the hydrocarbon chains. We find that dipole orientation does not show a strong dependence with temperature, at least, for the temperature range investigated here.

We have shown [20] that water confined in Newton Black Films exhibits an anomalous dielectric response. This response results from the polarization of the water molecules in the film, which is different from the polarization expected in an ordinary dielectric. We have proposed that this anomalous dielectric response is responsible for a non classical electrostatic repulsive force that has the same order of magnitude than the 'hydration force' measured in the experiments. The dipole orientational profiles in Figure 8 shows that water is polarized in a similar way for all the temperatures investigated. According to this we expect that the anomalous dielectric response will be present even at the highest temperature 
investigated here. We can gain a more quantitative understanding of the electrostatics of the Newton Black Films by computing the charge density profile and the associated electrostatic fields. Figure 9 shows the charge density profile for the Newton Black Film at ambient temperature. We have also represented the free charges (surfactants and counterions) and water contributions to the total charge distribution. Our results show that in the thin films the polarization of water results in charge separation. Thus, the water profile is characterized by positive contributions on the surfactant region, due to hydrogen partial charges, and a negative contribution in the middle of the film, due to a balance of the oxygen and hydrogen partial charges. The water charge density adds up to the free charge one to give a final charge density that is oscillating, reflecting the existence of alternating regions with charge of opposite sign. These oscillations are reflected in the associated electrostatic fields. The electrostatic field in the direction normal to the film plane can be obtained using Gauss's theorem [20, 34]:

$$
E_{z}=\frac{1}{\epsilon_{0}} \int_{-\infty}^{z} \rho\left(z^{\prime}\right) d z^{\prime}
$$

where $\rho\left(z^{\prime}\right)$ is the total charge density at position $z^{\prime}$. The total charge density has two contributions, $\rho\left(z^{\prime}\right)=\rho_{f}\left(z^{\prime}\right)+\rho_{\text {water }}\left(z^{\prime}\right)$; the first contribution corresponds to the " free" , charges (surfactants and counterions only) and the second to the partial charges in the SPC/E water molecule. By splitting the charge density in these two contribution we can calculate the fields associated to the free charges only,

$$
E_{\text {free charges }, z}=\frac{D_{z}}{\epsilon_{0}}=\frac{1}{\epsilon_{0}} \int_{-\infty}^{z} \rho_{f}\left(z^{\prime}\right) d z^{\prime},
$$

where $D_{z}$ is the electric displacement, and to the water molecules,

$$
E_{\mathrm{water}, z}=\frac{1}{\epsilon_{0}} \int_{-\infty}^{z} \rho_{\text {water }}\left(z^{\prime}\right) d z^{\prime}
$$

which is related to the polarization, $P_{z}$, through, $P_{z}=-\epsilon_{0} E_{\text {water }, z}$, or alternatively:

$$
P_{z}=D_{z}-\epsilon_{0} E_{z}
$$

Figures 10 a-c show the electrostatic field of the Newton Black Films for the three temperatures considered in this work. The main result is that water polarization induces in some regions of the film an electrostatic field that has a sign opposite to that of the field 
due to the free charges alone. In other words, in some parts of the film water produces an overscreening of the electric field generated by the ionic species. As a result of this partial overscreening, the charge profile and the total field have an oscillating nature. This behavior is qualitatively consistent with recent theories of nonlocal electrostatics [36], which predict oscillating electric fields near interfaces due to the anomalous dielectric behavior of interfacial water. It is also interesting to recall that the electrostatic field inside the film is zero only in the middle of the film, this being a trivial consequence of the symmetry of system. The temperature slightly modifies the strength of the field, which decreases with increasing temperature. At HIGH temperature there are also less well defined oscillations and it is observed a reduction in the maximum polarization attained by the water molecules (see maximum and minimum of the water electrostatic field). Nevertheless the polarization decreases only by $10 \%$ in going from the ambient to the HIGH temperature system. This observation implies that water is highly ordered in the thin film, even at $348 \mathrm{~K}$ where we found significant structural changes with respect to the ambient temperature system. The strong ordering of water in these films results in a breakdown of the local relation,

$$
P_{z}=\left(\epsilon_{r}-1\right) \epsilon_{0} E_{z}
$$

where $\epsilon_{r}$ is the water permittivity. This relation breaks down because it disregards the molecular nature of water and other effects that determine the final dielectric response of water. These effects are nonlocal in nature and preclude the use of eqn. (11), i.e., it is not possible to obtain a well-defined function $\epsilon_{r}$ relating the polarization and the total field. The so-called non-local electrostatic theories try to generalize eqn. (11) by using an integral equation that relates the polarization and the total field [36]. In these theories, the kernel of the integral equation plays the role of a generalized dielectric permittivity. Nonetheless, these theories are still controversial and do not provide a full explanation of the complex dielectric behavior of interfacial water.

One important mechanism underlying this complex response of interfacial water should be water hydrogen bonding, which can be very significant in determining the water structure at interfaces. As a matter of fact, water next to hydrophobic surfaces maximizes the number of hydrogen bonds per molecule by reorienting the water molecules and creating dangling $\mathrm{OH}$ bonds [33]. This complex interfacial behavior explains why theories such as the DLVO, which model water as a continuum dielectric medium are bound to fail in the description of 
highly confined systems.

\section{CONCLUSIONS}

Large scale molecular dynamics simulations of Newton Black Films of $3 \mathrm{~nm}$ thickness (4.4 water molecules per surfactant) have been presented. These films have a thickness that closely corresponds to the one measured in films formed spontaneously from surfactant solutions [7]. In this work we have investigated the temperature dependence of the structure and electrostatic properties of these films. With this purpose we have considered films at ambient temperature (298 K), LOW temperature (248 K) and HIGH temperature (348 $\mathrm{K})$. Our work shows that the structure of the ambient temperature film is preserved upon cooling. We do not observe major changes in the film fluctuations, and the roughness of both systems is essentially the same, despite the fact that in the LOW temperature systems there is a significant reduction in the thermal energy. On the other hand heating the Newton Black Film to high temperatures $(348 \mathrm{~K})$ results in a major disruption of the film internal structure, showing evidence for bilayer fusion. More importantly the roughness increases dramatically with temperature. This is expected considering that an increase in temperature enhances thermal fluctuations. Assuming that the increase in roughness with temperature can be assigned to thermal fluctuations, we estimate that the surface tension of the HIGH temperature system should decrease at least by a factor of 2 with respect to the surface tension at ambient temperature. We would like to emphasize that thermal fluctuations alone cannot explain the structural changes observed in the LOW temperature system. Our results suggest that thermal fluctuations might not be the only element defining the roughness and therefore the disorder of the film, even at ambient temperature. This might be relevant to ascertain whether entropic forces can really play a significant role in determining the stability of ionic Black Films. Otherwise we have shown that the structural changes described above take place with no noticeable change in the overall thickness of the film. This result is compatible with recent neutron scattering experiments of oil-SDSwater films, which also reported an insensitivity of the the overall thickness of the film to temperature [10].

One important feature of Newton Black Films is that water is strongly polarized. We observe that the film favors orientations where the water dipole is pointing toward the hy- 
drocarbon layers. The main consequence of this orientation is the charge separation in the water charge density profile. This charge separation combined with the surfactant and counterion charge contributions results in an oscillating charge density profile. This is reflected in the electrostatic fields that are also oscillatory. We find that the water orientation and polarization in the film exhibit very little dependence with temperature. Water molecules are highly ordered, even at the highest temperature investigated, $348 \mathrm{~K}$. This suggest that the anomalous dielectric behavior described by us [20] should also be significant at these high temperatures.

We expect that the results obtained in this work will be relevant to other systems such as, the smectic liquid crystals formed in lipid membranes, the Newton Black Films formed in emulsion droplets, and to understant and develop new microscopic models to interpret the experimental behavior of these fascinating systems.

\section{Acknowledgments}

FB would like to thank EPSRC (Research Grant No. GR/R39726/01) for funding. The simulations were performed at the HPCx supercomputing center (Edinburgh, UK) under the Materials Chemistry consortium (UK), and the Mare Nostrum Supercomputer (Barcelona Supercomputer Center, Spain).

[1] D. Evans and H. Wennerstrom, H. 'The Colloidal Domain'. 2nd Edition, Wiley-VCH: New York (1999).

[2] V. Bergeron. 'Forces and structure in thin liquid soap films'. J. Phys.: Condens Matter, 11, R215 (1999).

[3] C. Stubenrauch and R. von Klitzing. 'Disjoining pressure in thin liquid foam and emulsion filmsnew concepts and perspectives'. J. Phys.: Condens Matter, 15, R1197(2003).

[4] C. Hedreul and G. Frens. 'Foam stability'. Colloids and Surfaces A, 186, 73 (2001).

[5] E.S. Boek, A. Jusufi, H. Löwen, G.C. Maitland. 'Molecular design of responsive fluids: molecular dynamics studies of viscoelastic surfactant solutions '. J. Phys.: Condens. Matter, 14, 9413 (2002). 
[6] D. Exerova, T. Kolarov and KHR. Khristov. 'Direct Measurement of Disjoining Pressure in Black Foam Films I. Films from an ionic Surfactant'. Colloids and Surfaces, 22, 171 (1987).

[7] O. Belorgey and J. Benattar. 'Structural-properties of soap black films investigated by X-ray reflectivity'. Phys. Rev. Lett., 66, 313 (1991).

[8] V. Casteletto et. al. 'Stability of Soap Films: Hysteresis and nucelation of Black Films' Phys. Rev. Lett., 90, 048302 (2003).

[9] R. Krustev, H.J. Muller, and J.L. Toca-Herrea. 'The thickness and contact angle of sodium dodecyl sulfate foam films depending on the concentration of LiCl'. Colloid Polym. Sci., 276, 518 (1998).

[10] P. Poulin, F. Nallet, B. Cabane and J. Bibette. 'Evidence for Newton Black Films between adhesive emulsions droplets'. Phys. Rev. Lett., 77, 3248 (1996).

[11] T.J. McIntosh and S.A. Simon. 'Hydration force and bilayer deformation: A reevaluation'. Biochemistry, 25, 4058 (1986).

[12] S. Leikin, V.A. Parsegian and D.C. Rau and R.P. Rand. 'Hydration Forces'. Annu. Rev. Phys. Chem., 44, 369 (1993).

[13] S. Marcelja and N. Radic. 'Repulsion of Interfaces due to boundary water'. Chem. Phys. Lett., 42, 129 (1976).

[14] M. Manciu, and E. Ruckenstein. 'The polarization model for hydration/double layer interactions: the role of the electrolyte ions'. Adv. Coll. Interface Sci., 112, 109 (2004).

[15] J. Israelacjvili and H. Wennerstrom. 'Entropic Forces between Amphiphilic Surfaces in Liquids'. J. Phys. Chem., 96, 520 (1992).

[16] J. Israelachvili and H. Wennerstrom. 'Role of hydration and water structure in biological and colloidal interactions'. Nature (London), 379, 219 (1996).

[17] V.A. Parsegian and R.P. Rand. 'On Molecular Protrusion as thr Spource of Hydration Forces', Langmuir, 7, 1299 (1991).

[18] Z. Gamba, J. Hautman, J.C. Shelley, and M.L. Klein. 'Molecular-dynamics investigation of a Newton Black Film'. Langmuir, 8, 3155 (1992).

[19] F. Bresme and J. Faraudo. 'Computer Simulation Studies of Newton Black Films'. Langmuir, 20, 5127 (2004).

[20] J. Faraudo and F. Bresme. 'Anomalous dielectric behaviour of water in ionic Newton Black Films'. Phys. Rev. Lett., 92, 236102 (2004). 
[21] J. Faraudo and F. Bresme. 'Origin of the short-ranged, strong repulsive force between ionic surfactant layers'. Phys. Rev. Lett., 94, 077802 (2005).

[22] Forester, T.R., and Smith, W. 'DLPOLY 2 package of Molecular Simulation'. CCLRC, Daresbury Lab (2001).

[23] W.D. Cornell et al., 'A second generation force field for the simulation of proteins, nucleic acids and organic molecules'. J. Am. Chem. Soc., 117, 5179 (1995).

[24] K.J. Schweighofer, U. Essmann, and M. Berkowitz. 'Simulation of sodium dodecyl sulfate at the water-vapor and water-carbon tetrachloride interfaces at low surface coverage'. J. Phys. Chem., 101, 3793 (1997); H. Dominguez, M.L. Berkowitz. 'Computer simulations of sodium dodecyl sulfate at liquid/liquid and liquid/vapor interfaces'. J. Phys. Chem. B, 104, 5302 (2000).

[25] J. Shelley, K. Watanabe and M.L. Klein. 'Simulation of a sodium dodecyl-sulfate micelle in aqueous solution'. Int. J. Quantum Chem.: Quantum Biol. Symp., 17, 103 (1990).

[26] H.J. Berendsen, J. Grigera and T.P. Straatsma, 'The missing term in effective pair potentials'. J. Phys. Chem., 91, 6269 (1987).

[27] A. Wallqvist. 'Polarizable water at a hydrophobic wall'. Chem. Phys. Lett., 165, 437 (1990).

[28] I. Yeh and M.L. Berkowitz. 'Effects of the polarizability and water density constraint on the structure of water near charged surfaces: Molecular dynamics simulations'. J. Chem. Phys., 111, $10491(2000)$.

[29] I. Yeh and M.L. Berkowitz. 'Ewald summation for systems with slab geometry'. J. Chem. Phys., 111, 3155 (1999).

[30] W.L. Jorgensen and J. Tirado-Rives. 'Potential energy functions for atomic-level simulations of water and organic and biomolecular systems'. Proc. Nat. Acad. Sci. USA, 102, 6665. (2005).

[31] E. Sanz, C. Vega, J. L. F. Abascal, and L. G. MacDowell. 'Phase Diagram of Water from Computer Simulation'. Phys. Rev. Lett., 92, 255701 (2004).

[32] R.R. Netz. 'Electrostatics of counter-ions at and between planar charged walls: From PoissonBoltzmann to the strong-coupling theory'. Eur. Phys. J. E, 5557 (2001).

[33] K. Tay and F. Bresme. 'Hydrogen bond structure and vibrational spectrum of water at a passivated metal nanoparticle'. J. Mater. Chem., 10.1039/b600252h (2006).

[34] J.D. Jackson. 'Classical Electrodynamics'. 3rd ed. (Wiley, New York) (1999).

[35] L.X. Dang. 'Mechanism and thermodynamics of ion selectivity in aqueous solutions of 18- 


\section{Page 19 of 36}

1

2

3

4

5

6

7

8

9

10

11

12

13

14

15

16

17

18

19

20

21

22

23

24

25

26

27

28

29

30

31

32

33

34

35

36

37

38

39

40

41

42

43

44

45

46

47

48

49

50

51

52

53

54

55

56

57

58

59

60

crown-6 ether - A molecular dynamics stuy'. J. Am. Chem. Soc., 117, 6954 (1995).

[36] D. A. Cherepanov. 'Force Oscillations and Dielectric Overscreening of Interfacial Water'. Phys. Rev. Lett., 93, 266104 (2004). 
TABLE I: Bonding interactions. Energies are given in $\mathrm{kJ} / \mathrm{mol}$ and distances in $\AA$.

\begin{tabular}{|c|c|}
\hline nte & Interaction Parameters Ref \\
\hline bond $\mathrm{CH}_{3}-\mathrm{CH}_{2}$ & $k_{b}=2594.1, r_{e}=1.526 \AA[23]$ \\
\hline bond $\mathrm{CH}_{2}-\mathrm{CH}_{2}$ & $k_{b}=2594.1, r_{e}=1.526 \AA[23]$ \\
\hline bond $\mathrm{CH}_{2}-\mathrm{O}$ (ester) & $k_{b}=2677.8, r_{e}=1.410 \AA[23]$ \\
\hline bond $\mathrm{O}$ (ester)-S & $k_{b}=2677.8, r_{e}=1.580 \AA[23]$ \\
\hline bond S-O & $k_{b}=7531.2, r_{e}=1.460 \AA[24]$ \\
\hline bending $\mathrm{CH}_{3}-\mathrm{CH}_{2}-\mathrm{CH}_{2}$ & $k_{i j k}=334.72, \theta_{e}=109.5^{\circ}[23]$ \\
\hline bending $\mathrm{CH}_{2}-\mathrm{CH}_{2}-\mathrm{CH}_{2}$ & $k_{i j k}=334.72, \theta_{e}=109.5^{\circ}[23]$ \\
\hline bending $\mathrm{CH}_{2}-\mathrm{CH}_{2}-\mathrm{O}$ (ester) & $k_{i j k}=418.40, \theta_{e}=109.5^{\circ}[23]$ \\
\hline bending $\mathrm{CH}_{2}-\mathrm{O}$ (ester)-S & $k_{i j k}=418.40, \theta_{e}=112.6^{o}[23]$ \\
\hline bending $\mathrm{O}$ (ester)-S-O & $k_{i j k}=427.00, \theta_{e}=102.6^{\circ}[23]$ \\
\hline bending $\mathrm{O}-\mathrm{S}-\mathrm{O}$ & $k_{i j k}=427.00, \theta_{e}=115.4^{o}[23]$ \\
\hline dihedral $\mathrm{CH}_{3}-\mathrm{CH}_{2}-\mathrm{CH}_{2}-\mathrm{CH}_{2}$ & $A_{i j k l}=11.7152$ \\
\hline dihedral $\mathrm{CH}_{2}-\mathrm{CH}_{2}-\mathrm{CH}_{2}-\mathrm{CH}_{2}$ & $A_{i j k l}=11.7152$ \\
\hline dihedral $\mathrm{CH}_{2}-\mathrm{CH}_{2}-\mathrm{CH}_{2}-\mathrm{O}$ (ester) & $A_{i j k l}=11.7152$ \\
\hline dihedral $\mathrm{CH}_{2}-\mathrm{CH}_{2}-\mathrm{O}$ (ester)-S & $A_{i j k l}=9.6232$ \\
\hline dihedral $\mathrm{CH}_{2}-\mathrm{O}$ (ester)-S-O & $A_{i j k l}=2.0920$ \\
\hline
\end{tabular}


TABLE II: Non bonding interactions. Parameters for Lennard Jones interactions and partial charges $\left(q_{e}\right.$ is the absolute value of the electronic charge)

\begin{tabular}{lcccc} 
Group & $\epsilon(\mathrm{KJ} / \mathrm{mol})$ & $\sigma(\AA)$ & $q / q_{e}$ & Ref \\
\hline $\mathrm{Na}$ & 0.4179 & 2.5840 & 1.0 & {$[35]$} \\
$\mathrm{O}$ (water) & 0.6502 & 3.1660 & -0.8476 & {$[26]$} \\
$\mathrm{H}$ (water) & 0 & 0 & 0.4238 & {$[26]$} \\
$\mathrm{CH}_{3}$ & 0.4577 & 3.816 & 0 & {$[23]$} \\
$\mathrm{CH}_{2}$ & 0.4577 & 3.816 & 0 & {$[23]$} \\
$\mathrm{CH}_{2}$ bonded & 0.4577 & 3.816 & 0.137 & {$[23]$} \\
to O(ester) & & & & \\
$\mathrm{O}$ (ester) & 0.7133 & 3.3224 & -0.459 & {$[23]$} \\
$\mathrm{S}$ & 1.0460 & 4.0000 & 1.284 & {$[23]$} \\
$\mathrm{O}$ & 0.8786 & 3.3224 & -0.654 & {$[23]$} \\
\hline
\end{tabular}

FIG. 1: Snapshot of a representative sodium dodecyl sulfate Newton Black Film (SDS-NBF) containing 512 surfactants, and 6112 water molecules.

FIG. 2: The dodecyl sulfate surfactant. The 'all atom' (top) and the united atom models (bottom). The partial charges defining the charge distribution of the sulfate head group are also shown. 
FIG. 3: Log-log plot showing the scaling of the CPU time per step with the number of processors for two representative Newton Black Films. The tests were performed with the code DLPOLY 2 in the Mare Nostrum Supercomputer (Spain).

FIG. 4: Representative snapshot of the SDS-NBF (4.4 water molecules per surfactant) at a) 298 $\mathrm{K}$ and b) $348 \mathrm{~K}$. Water is not shown for the sake of clarity. Surfactants are represented as bonds and $\mathrm{Na}^{+}$cations as spheres.

FIG. 5: a) Density profiles across the SDS-NBF for three different temperatures. Full lines, $\mathrm{CH}_{3}$ pseudoatoms, dashed line, sulfur atom and dashed-dotted lines sodium cations. b) Oxygen density profile across the Newton Black Film as a function of temperatures, $348 \mathrm{~K}$ (full line), $298 \mathrm{~K}$ (dashed line) and $248 \mathrm{~K}$ (dash-dotted line).

FIG. 6: Temperarure dependence of the SDS-NBF roughness (eqn. (4)). Full line, 348 K, dashed line, $298 \mathrm{~K}$ and dash-dotted line $248 \mathrm{~K}$.

FIG. 7: Electron density profile of the Newton Black Film as a function of temperature. The experimental electron density profile is also shown [7].

FIG. 8: The orientation of the water molecules across the Newton Black Film for different temperatures. The density profile for the sulfur atoms (dotted line) at $298 \mathrm{~K}$ is also shown for comparison.

FIG. 9: The charge density profile of the SDS-NBF at ambient temperature. The free charges and water contributions to the total charge density have been included for comparison. See text for details.

FIG. 10: Electrostatic field (full line) and electrostatic field contributions due to the free charges (dashed line) and water (dash-dotted line) at different temperatures. a) $348 \mathrm{~K}$, b) $298 \mathrm{~K}$ and c) $248 \mathrm{~K}$. 


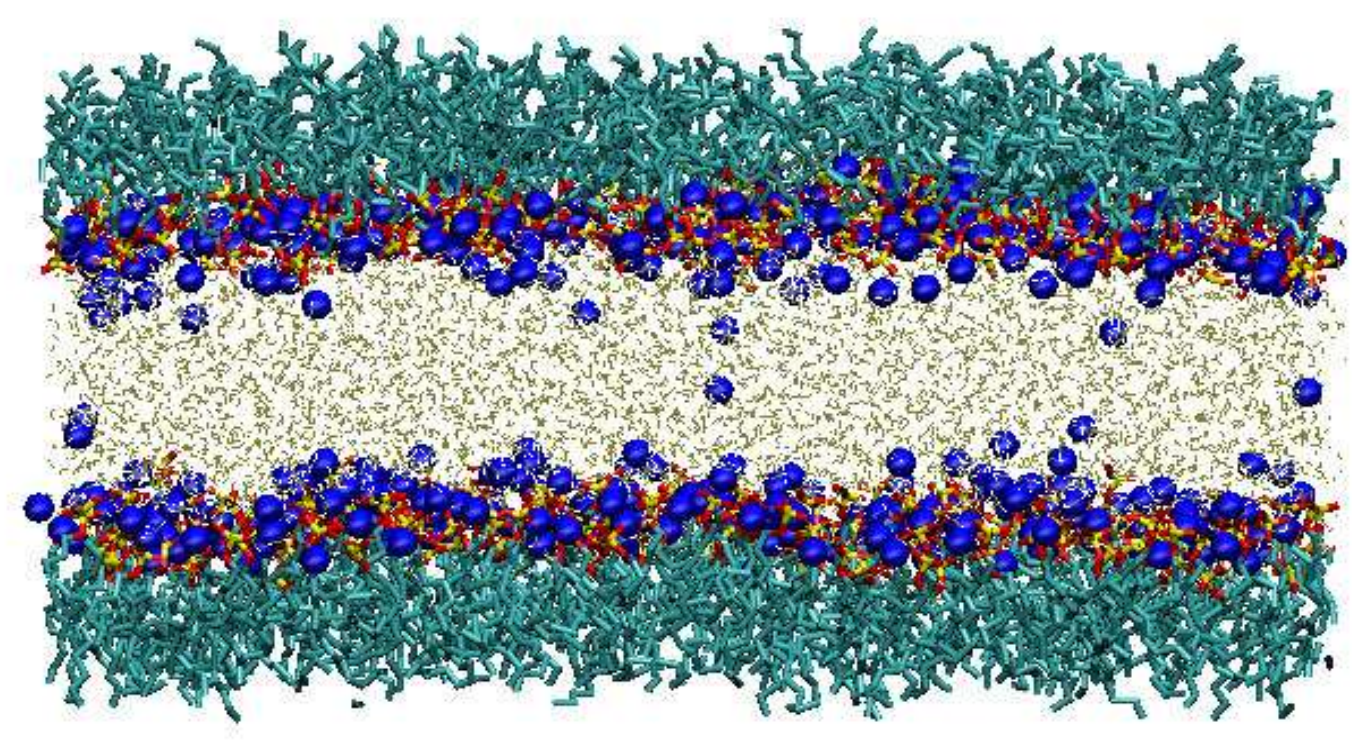

Fig 1: Bresme and Faraudo 

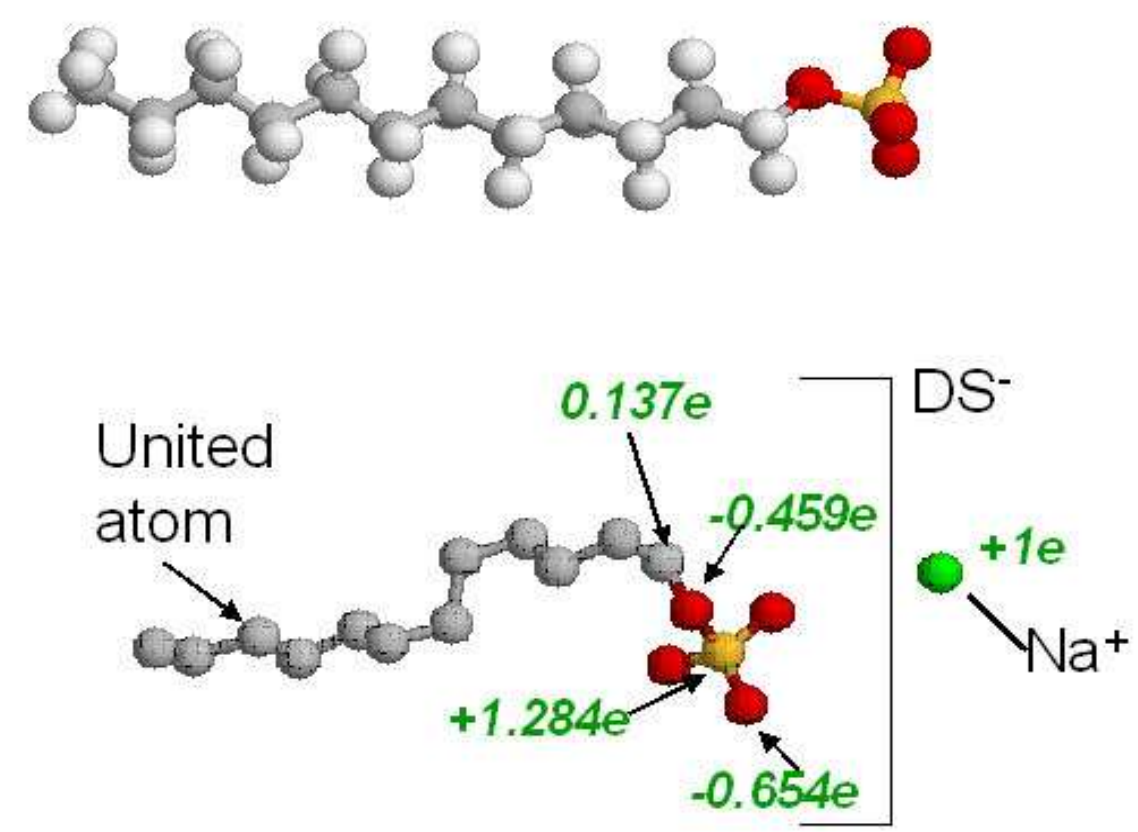

Fig 2: Bresme \& Faraudo 


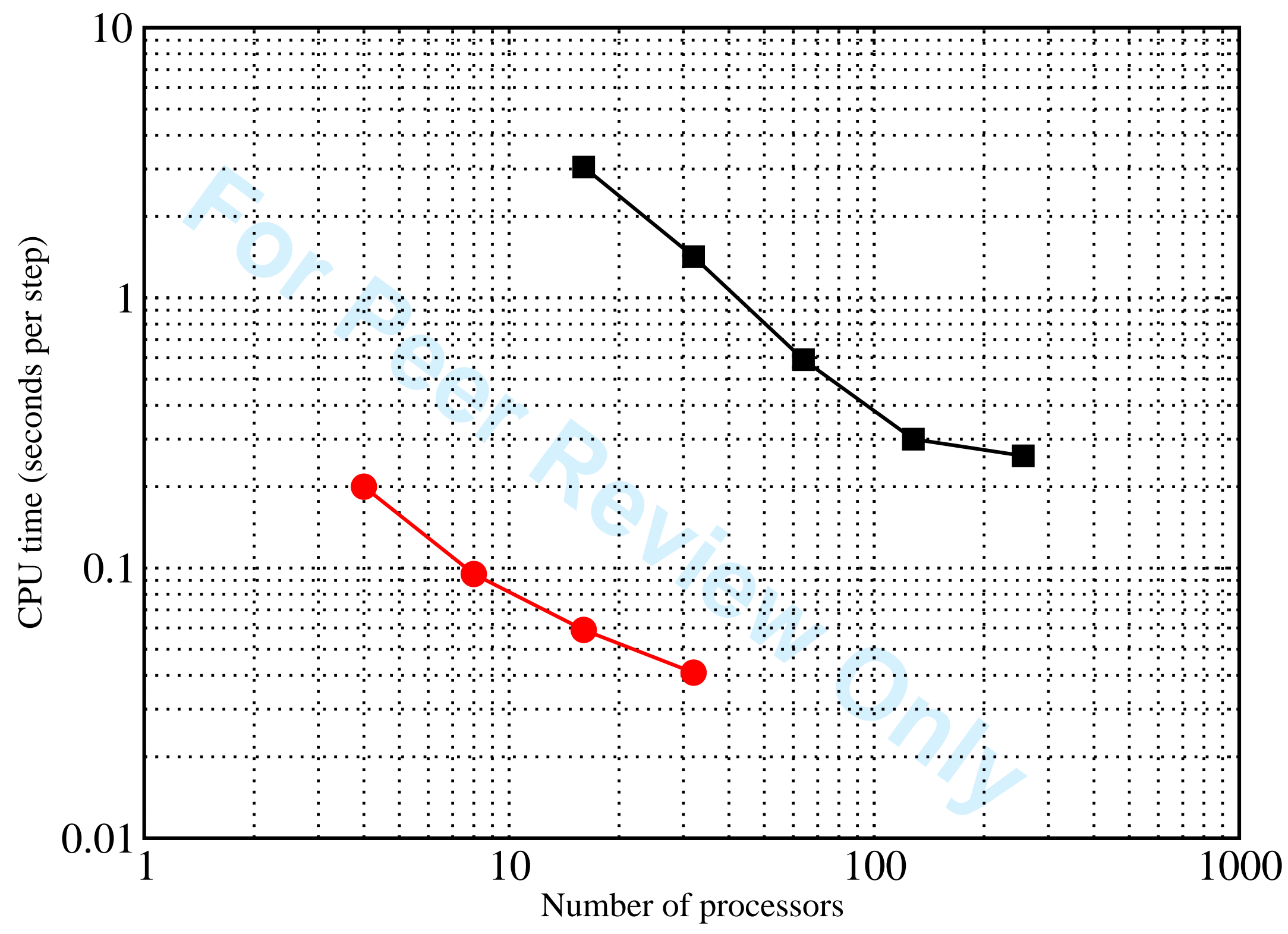

Fig 3: Bresme and Faraudo 


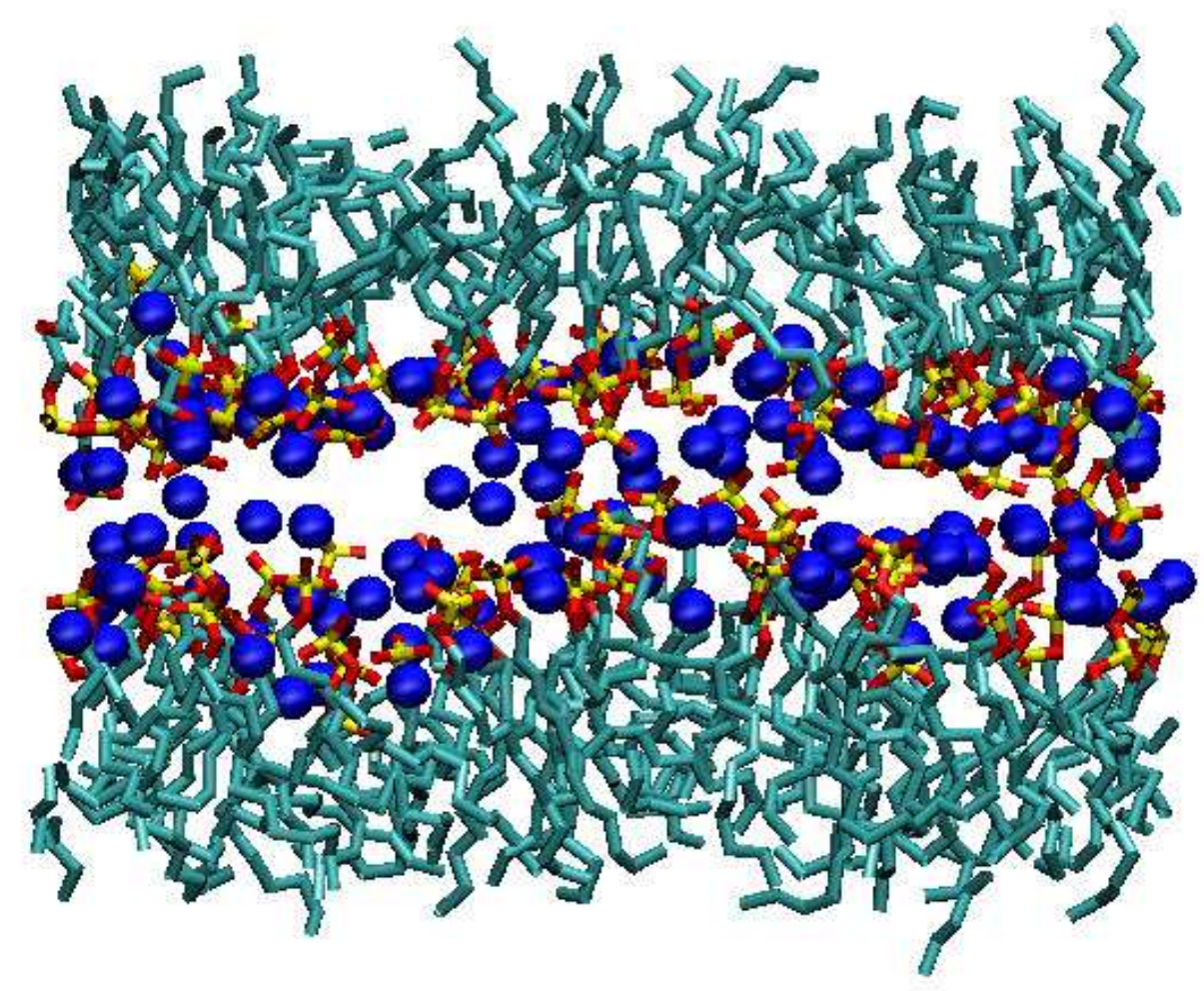

Fig 4 a: Bresme and Faraudo 


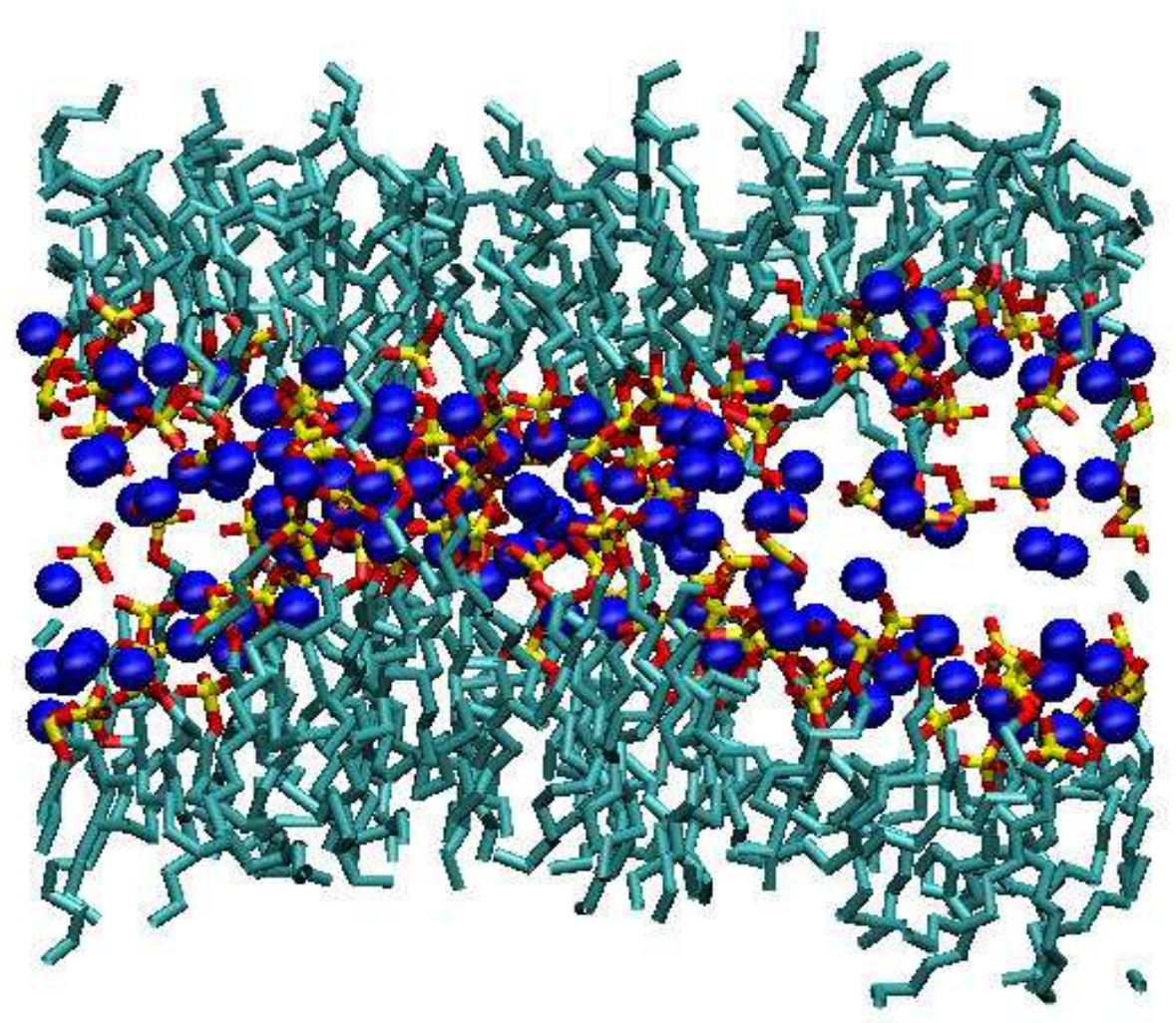

Fig 4b: Bresme and Faraudo 


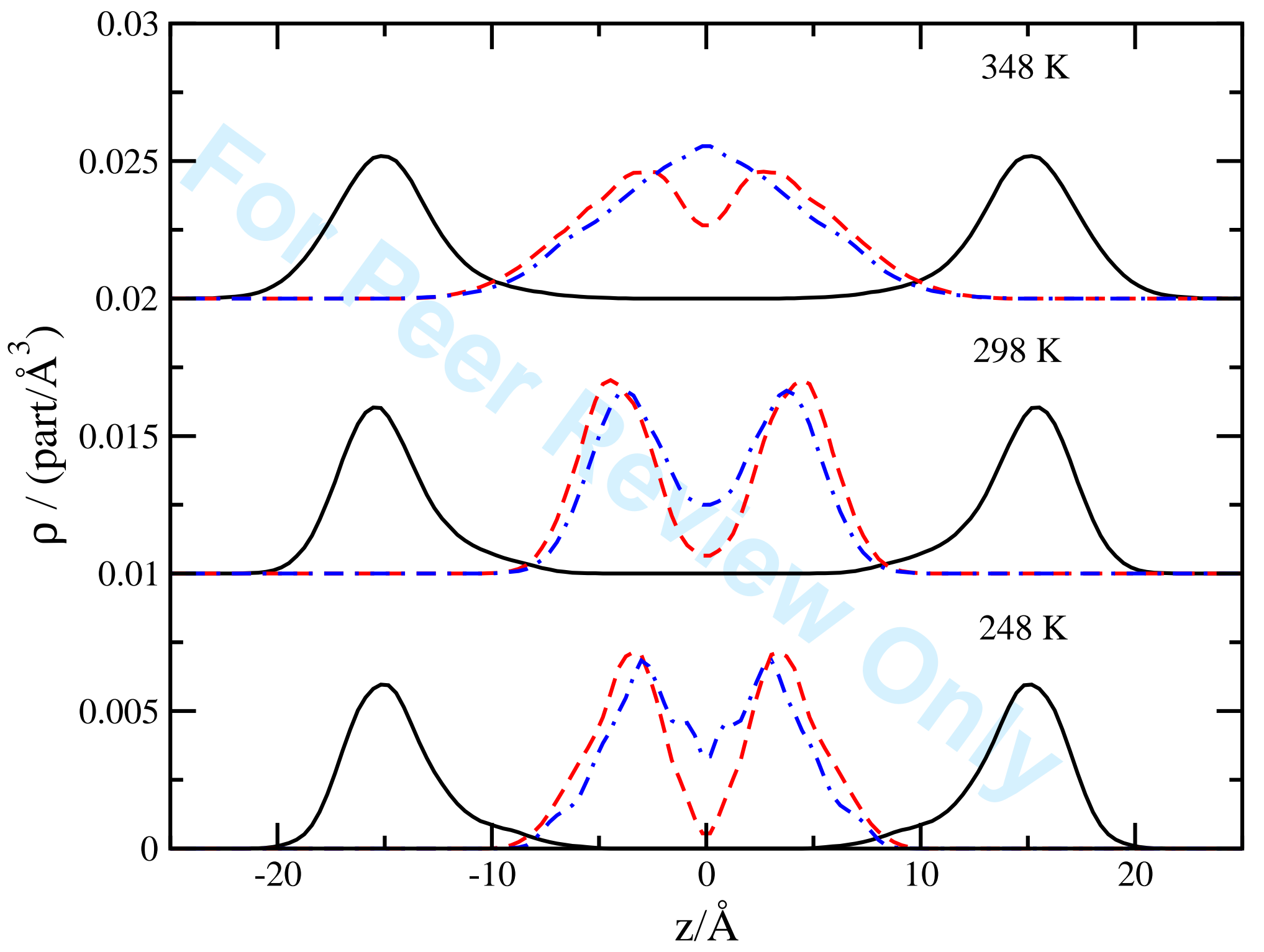

Fig 5a: Bresme and Faraudo 


\section{Page 29 of 36}

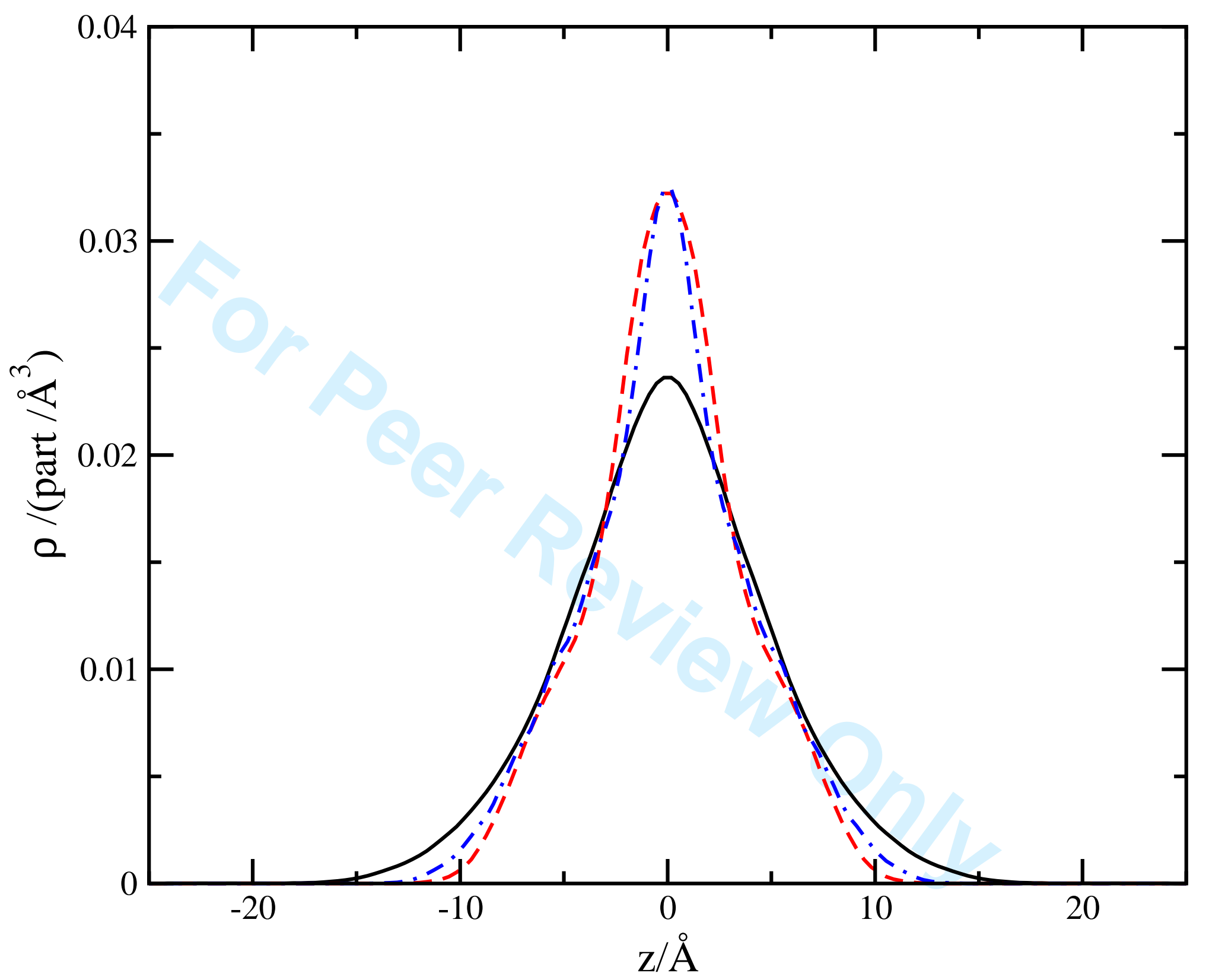

Fig 5b: Bresme and Faraudo 


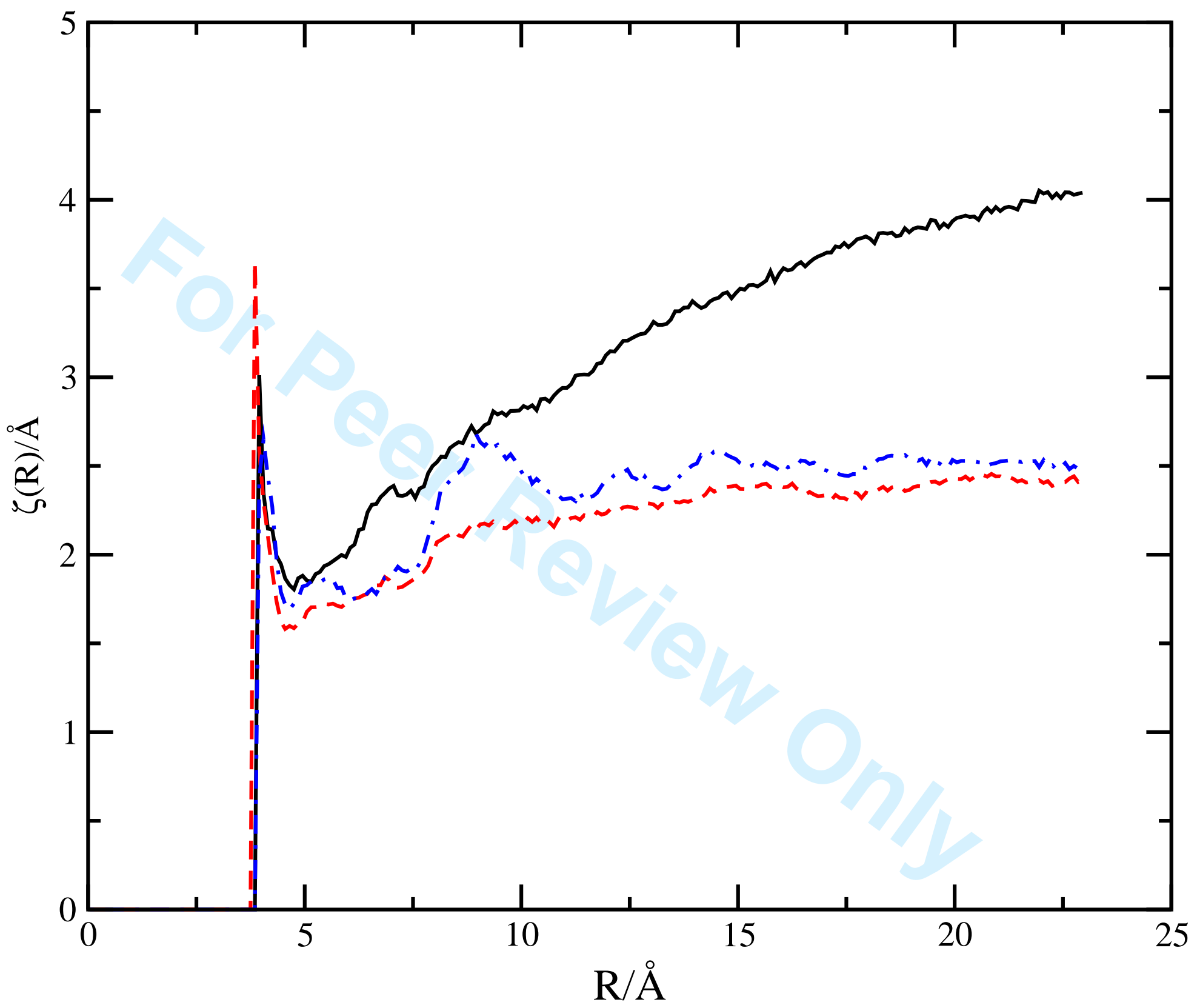

Fig 6: Bresme and Faraudo

http://mc.manuscriptcentral.com/tandf/jenmol 


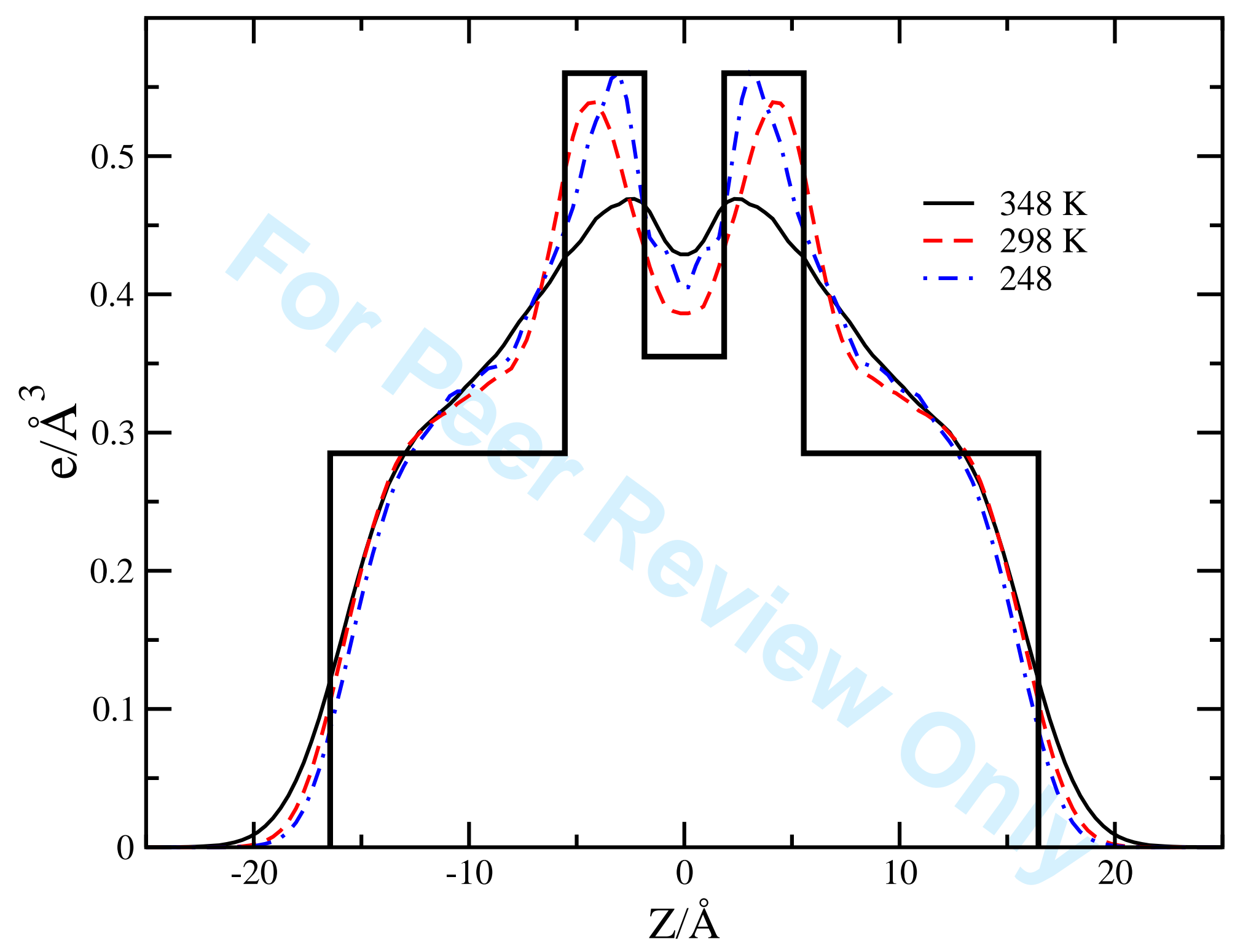

Fig 7: Bresme and Faraudo 


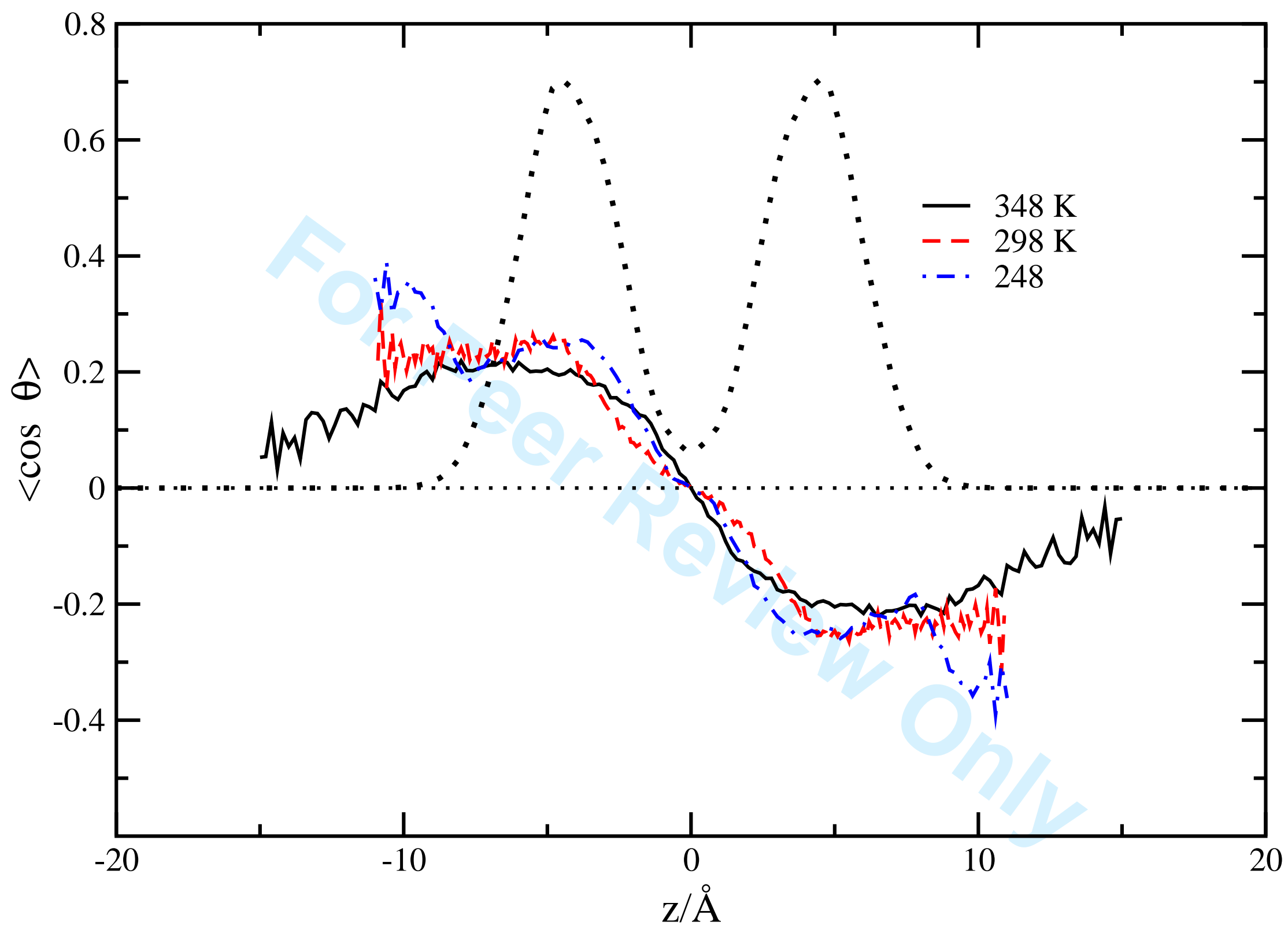

Fig 8: Bresme and Faraudo 


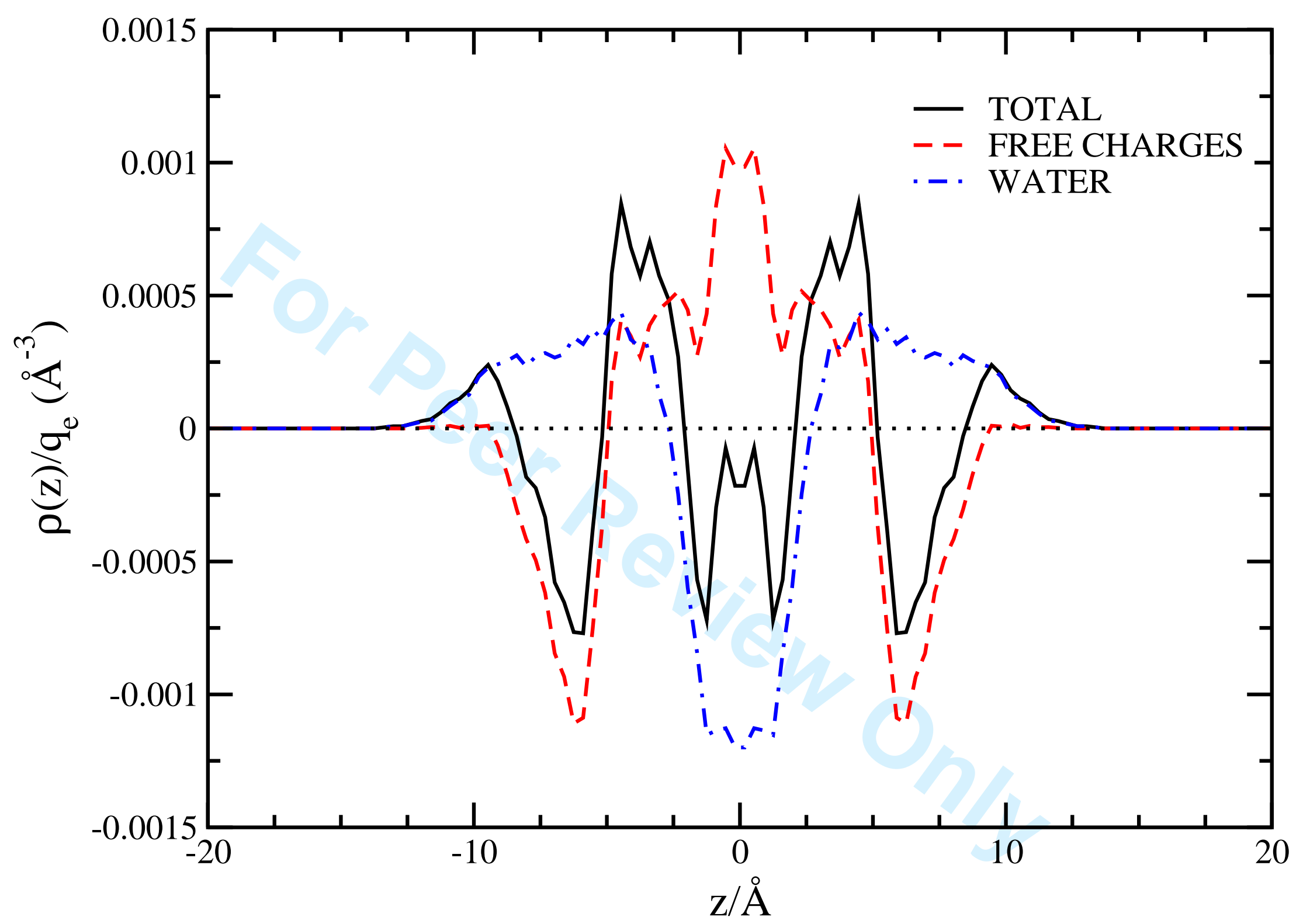

Fig 9: Bresme and Faraudo 


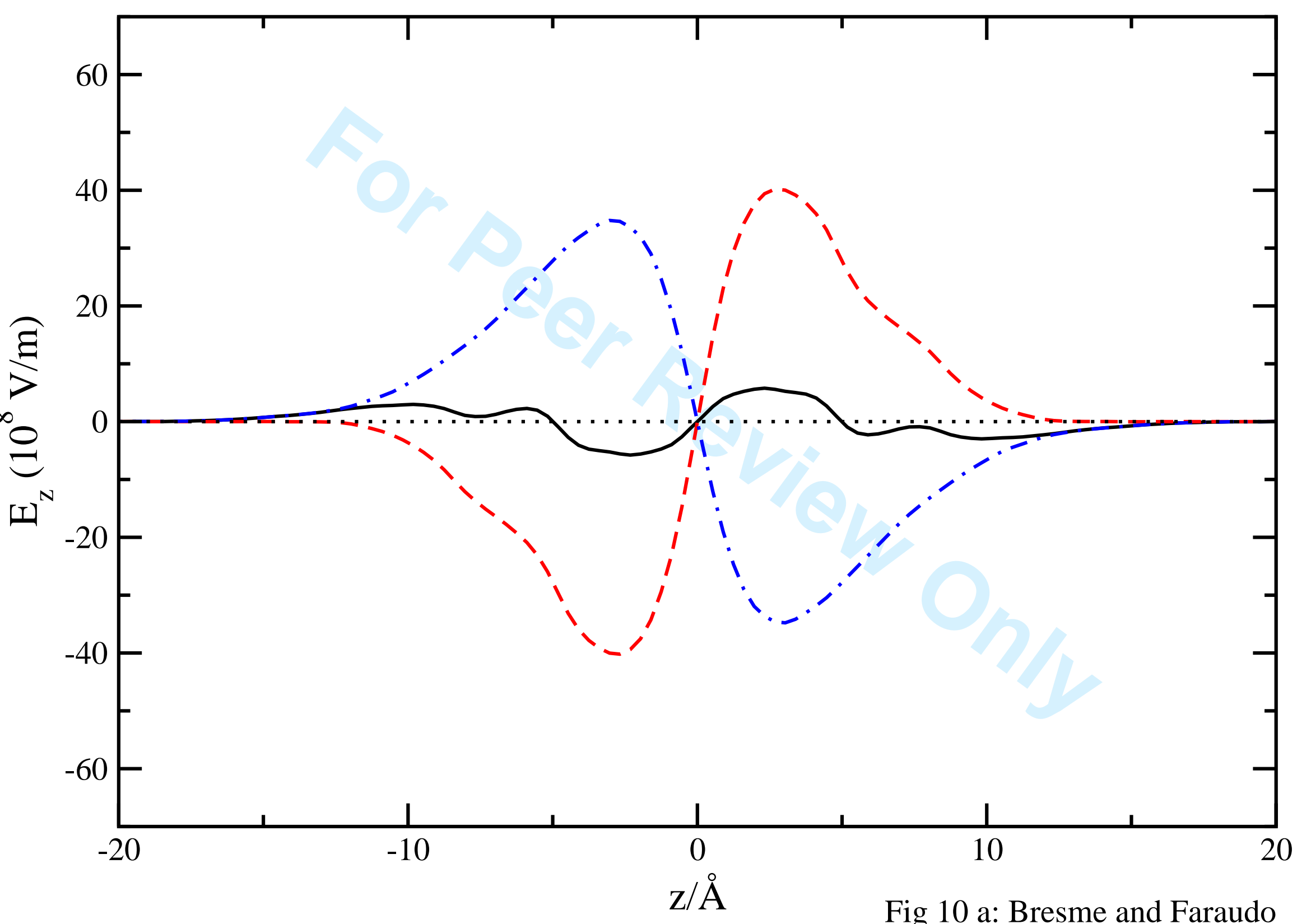

http://mc.manuscriptcentral.com/tandf/jenmol 


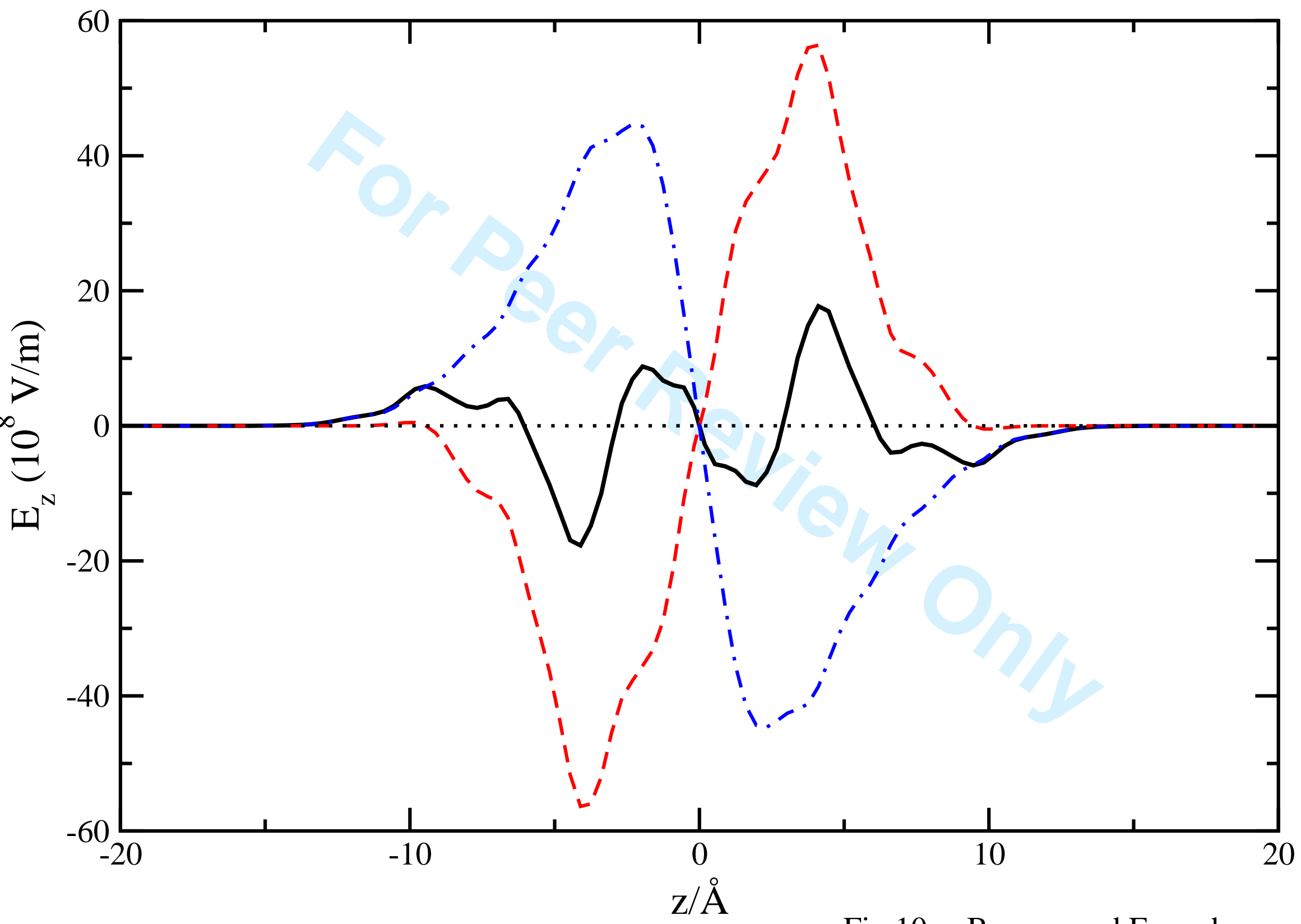

Fig 10 c: Bresme and Faraudo http://mc.manuscriptcentral.com/tandf/jenmol 